\title{
KRAS mutations and subtyping in colorectal cancer in Jordanian patients
}

\author{
WAFA M. ELBJEIRAMI and MAHER A. SUGHAYER \\ Department of Pathology and Laboratory Medicine, King Hussein Cancer Center, Amman 11941, Jordan
}

Received April 18, 2012; Accepted May 30, 2012

DOI: $10.3892 / \mathrm{ol} .2012 .785$

\begin{abstract}
Colorectal cancer (CRC) is one of the most common malignancies in the Western world and Jordan. v-Ki-ras2 Kirsten rat sarcoma (KRAS) mutations represent an early event in the development and progression of CRC. Previous studies have demonstrated that KRAS mutations serve as a predictor of response to EGFR-targeted therapies for patients with metastatic CRC. The aim of this study was to determine the portion of CRC patients with wild-type KRAS status and molecular subtypes of KRAS mutations in Jordan as compared with other countries. DNA was isolated from 100 consecutive colorectal carcinoma specimens from patients who underwent surgical resection or colonoscopic biopsies of colorectal tumors and had developed metastatic disease. KRAS mutations were detected by hybridization-based strip assay as well as RT-PCR-based assay and confirmed by standard Sanger sequencing of codon 12 and 13 of exon 1 of the KRAS gene. Among 100 tested patients, 56\% had a wt-KRAS genotype and $44 \%$ had a mutated KRAS genotype. The pGly12Asp was the most commonly detected mutation (54.5\%). KRAS mutations were independently associated with patient age, gender and tumoral variables. The ratio of mutated versus wt-KRAS patients in this study is similar to those reported in Western countries but contrasts to neighboring Middle Eastern countries. Colorectal carcinoma cases from Jordan had higher $K R A S$ mutation frequencies compared with other Middle Eastern countries which is likely to reflect different molecular pathogenesis and environmental exposures.
\end{abstract}

\section{Introduction}

Colorectal cancer (CRC) is the second most common form of cancer in developed countries, only surpassed by prostate cancer in men and breast cancer in women (1). In Jordan, it is the most common type of cancer among men and the second

Correspondence to: Dr Maher A. Sughayer, King Hussein Cancer Center, Queen Rania Al Abdullah Street, P.O. Box 1269, Al-Jubeiha, Amman 11941, Jordan

E-mail: msughayer@khcc.jo

Key words: colorectal carcinoma, KRAS gene, mutation, Jordan most common among women (2). The reasons for this are unknown and may include both genetic and environmental factors. CRC can be cured by relatively simple colorectal procedures if detected early. However, distant metastasis is the main cause of mortality in CRC patients. Studies have shown that depending on the stage of the primary tumor, liver metastases occur in $20-70 \%$ of patients and lung metastases occur in $10-20 \%$ of patients (3).

Significant advances have been made in the treatment and outcome of CRC over the last decade. An improved understanding of the molecular pathways involved in the development and progression of CRC has made it possible to provide prognoses for patients with metastases, as well as the development of new therapeutic strategies. The epidermal growth factor receptor (EGFR) is a transmembrane tyrosine kinase receptor. It is expressed in epithelial tissues and acts as a cell growth promoter. According to literature, EGFR contributes to the development and progression of several types of cancer, including CRC where it is overexpressed in 50-80\% of colorectal tumors, making it a suitable target for anticancer therapies (4). Abnormal activation of the EGFR pathway may be caused by EGFR overexpression or mutational activation of the downstream elements (5).

Currently, two strategies to attenuate EGFR signaling are in use: monoclonal antibodies that bind to the ligand-binding domain and inhibit the binding of specific ligands (cetuximab and panitumumab), or small EGFR tyrosine kinase inhibitor molecules that bind to the intracellular domain of EGFR and compete for binding with ATP, inhibiting tyrosine phosphorylation (gefitinib and erlotinib). These inhibitors of EGFR have emerged as an important treatment for metastatic colorectal cancer (mCRC) (6,7). To optimize the benefits and reduce the risks of anti-EGFR therapies, EGFR as well as the molecules involved in its signaling pathway have been evaluated as potential markers for predicting therapy outcomes. Anti-EGFR therapies are only effective in a subset of patients with CRC. A number of clinical trials have demonstrated that EGFR-targeted therapies are not effective in patients whose tumors have a mutation in the oncogene Kirsten ras (KRAS) (8-10).

The v-Ki-ras2 Kirsten rat sarcoma $(K R A S)$ gene is a member of the Ras gene family that encodes small $\mathrm{G}$ proteins with intrinsic GTPase activity. KRAS is a downstream component of the EGFR signaling pathway. It acts as an intracellular signal transducer by coupling the signal from the 
cell surface receptors to intracellular targets, which regulate significant functions for tumor progression, including proliferation, differentiation and apoptosis (4). Mutations in the $K R A S$ gene (typically point mutations) result in constitutive guanine triphosphotase activity, which continuously activates signaling pathways in the absence of any upstream stimulation of EGFR/HER receptors (11). Thus, patients with KRAS mutations have poor responses to therapy with anti-EGFR inhibitors. KRAS mutations are thought to be a fairly early event in carcinogenesis and range from $35-45 \%$ in CRC (12). $K R A S$ mutations also occur frequently in non-small-cell lung and pancreatic carcinomas (13). The most common mutations identified in CRC occur in exon 2 and to a lesser extent in exon 3 (14). Tumors that have a mutation in codon 12 or 13 of the KRAS gene will not respond to treatment with EGFR inhibitors, including cetuximab or panitumumab.

The mutation status of the KRAS gene provides diagnostic, prognostic and predictive information for several types of cancer. The precise frequency and genotyping of KRAS mutations in the Jordanian population have not been determined. The high incidence and mortality among CRC Jordanian patients indicates the need to determine whether specific ethnic, geographical, dietary or lifestyle factors may possibly be correlated. This study investigated the general incidence of $K R A S$ mutations in CRC in Jordan and the incidence of specific mutation types. The possible correlation of molecular results with clinical and histopathological data was also analyzed.

\section{Materials and methods}

Ethics statement. This was an observational study. All patients were managed in accordance with normal clinical practice. The Institutional Review Board at King Hussein Cancer Center in Amman, Jordan, approved the current study.

Tissue attainment. Colorectal carcinoma specimens from 100 consecutive patients who underwent surgical resection or colonoscopic biopsies of colorectal tumors and had developed metastatic disease were studied. Biopsies or resected tumors were reviewed for their histological diagnosis and quantification of neoplastic cellularity $(>20 \%)$. Using hematoxylin and eosin-stained slides, areas with $>50 \%$ tumor cells were delimited.

All tumors were histologically confirmed to be colorectal adenocarcinomas. In addition, medical records were reviewed for information on the tumor site, pathological stage, presence or absence of metastasis and outcome in patients prior to anti-EGFR therapy. The formalin-fixed paraffin-embedded (FFPE) tissues were previously processed according to routine practices.

DNA extraction. DNA was extracted from paraffin blocks that best represented each tumor, previously selected from hematoxylin-eosin stained slides. To prevent cross contamination from tissues with flakes of paraffin, disposable scalpel blades were used. Tumor areas were carefully scraped from tissue blocks by macro-dissection using a sterile scalpel blade and then transferred to a microcentrifuge tube. Tissues were deparaffinized with three baths of xylene for 10 minutes followed by three baths of $100 \%$ ethanol solution for 5 minutes. Following this, tissues were digested with Proteinase $\mathrm{K}$ and genomic DNA was isolated using the QIAamp DNA Extraction kit (Qiagen, Crawley, UK) according to the manufacturer's instructions.

Samples of isolated genomic DNA were analysed by $0.8 \%$ agarose gel electrophoresis to evaluate the DNA quality. The DNA quantity was assessed by using NanoDrop 1000 (Thermo Scientific, DE, USA) and the purity was evaluated by calculating the 260/280 ratio.

KRAS mutational analysis. KRAS mutations were detected by an hybridization-based strip assay (ViennaLab ${ }^{\circledR}$ Diagnostics $\mathrm{GmbH}$, Vienna, Austria) as well as a real-time PCR-based mutation assay (DxS KRAS Mutation Test kit, DxS Ltd; Manchester, UK) according to the manufacturer's instructions. The first assay is based on reverse-hybridization of biotinylated PCR products to a parallel array of allele-specific oligonucleotides immobilized on membrane strips. The detection of specifically bound mutant $K R A S$ alleles is visible by an enzymatic color reaction which can be compared to specific controls. This assay detects 10 mutations located in codons 12 and 13. The second assay designed by DxS Diagnostic Innovations, combines allele-specific PCR Amplification Refractory Mutation System (ARMS) with real-time PCR to detect the seven most common mutations at KRAS codons 12 and 13 (p.Gly12Ala, p.Gly12Asp, p.Gly12Arg, p.Gly12Cys, p.Gly12Ser, p.Gly12Val and p.Gly13Asp). Mutation detection was performed with a Rotor Gene Q Real-Time PCR System (Corbett Robotics, Brisbane, Australia).

Random samples were selected for confirmation by standard Sanger sequencing using BigDye ${ }^{\circledR}$ terminator v3.1 (Applied Biosystems, Foster City, CA, USA). PCR was performed to amplify codons 12 and 13 of exon 1 in KRAS using specific primers under the PCR conditions described previously (15). The efficiency and quality of the amplification PCR were confirmed by running the PCR products on a $2 \%$ agarose gel. A negative control containing all the components of the PCR except the template was included in each PCR. DNA amplified products were purified using a QIAquick DNA clean up kit (Qiagen), according to the manufacturer's instructions. Amplification products were subjected to direct sequencing using the same primers and all mutations were confirmed by sequences originating from both the upstream and downstream primers on ABI 3130 Genetic Analyser (Applied Biosystems, Foster City, CA, USA). The presence of a mutation was accepted when its chromatographic peak height was $25 \%$ or higher than the peak of the wild-type reference.

Statistical differences were analyzed using a student's t-test and $\mathrm{P}<0.05$ was considered to indicate a statistically significant difference.

\section{Results}

Patient characteristics. Specimens from 100 tumors were retrospectively analyzed for the presence of KRAS mutations in codons 12 and 13 . The study included almost equal numbers of males and females (Table I). The median age at diagnosis was 55 years. The most common metastatic site was the liver (70\% of patients). The primary tumor site was the colon NOS (not otherwise specified) in 58\% of patients, the rectum in $22 \%$ and $20 \%$ were considered to be rectosigmoidal. All cancers 
Table I. Characteristics of the 100 patients enrolled in the study.

\begin{tabular}{lc}
\hline Characteristic & Value \\
\hline Total number of tumors & 100 \\
Gender & \\
Female & 45 \\
Male & 55 \\
Median age at diagnosis (range) & (22-74 years) \\
Primary tumor site & \\
Colon, NOS & 58 \\
Rectum & 22 \\
Rectosigmoid & 20 \\
Metastatic site at diagnosis & \\
Liver & 70 \\
Lung & 17 \\
None & 13 \\
TNM stage at diagnosis & \\
1 & 87 \\
2 & \\
3 & 5 \\
4 & \\
\hline
\end{tabular}

NOS, not otherwise specified.

were adenocarcinomas and were graded according to WHO criteria.

Prevalence of KRAS subtype mutations in Jordan. Of the 100 tumors included in this study, 44\% harbored KRAS mutations in either codon 12 or 13 (Fig. 1). Of the majority of KRAS mutations, 39 (89\%) were identified in codon 12, while codon 13 was involved in 5 (11\%) tumors (Fig. 2). Of the 39 mutations in codon 12 (wild-type GGT), 25 (62.5\%) were transition mutations, of which GAT (55\%) was the most common and $15(37.5 \%)$ were transversion mutations, of which GTT (14\%) was the most frequent; in codon 13 (wild-type GGC), only GAC transitions were present (Fig. 3). In one tumor, two mutations were identified in codon 12 , each with a transition and transversion mutational type (Gly to Asp and Gly to Cys). Several positive samples were randomly selected to confirm the detected mutation(s) by sequencing. A summary of all molecular types of KRAS mutations is shown in Table II.

Correlation of molecular findings with clinical and demographic data. KRAS mutations (codon 12 or 13) did not show any significant correlation with tumor location, stage, age at onset or gender of the patient (Table III).

KRAS mutations and prognosis. The response to standard therapy was documented for only 51 patients in the KHCC Cancer Registry. This is a small pool of data; however, findings are shared even if statistical analysis was challenging. In the distribution of patients with either wild-type or mutated $K R A S$, there is no association between response to therapy
Table II. Spectrum of KRAS mutations in 100 colorectal cancers.

\begin{tabular}{lrr}
\hline KRAS subtype mutation & No. & $\%$ \\
\hline pGly12Asp; codon 12 GGT>GAT & 24 & 54.5 \\
pGly12 Val; codon 12 GGT>GTT & 6 & 13.6 \\
pGly12Cys; codon 12 GGT>TGT & 5 & 11.4 \\
pGly12Ala; codon 12 GGT>GCT & 2 & 4.5 \\
pGly12Arg; codon 12 GGT>CGT & 2 & 4.5 \\
pGly12Ser; codon 12 GGT>AGT & 1 & 2.3 \\
pGly12Leu; codon 12 GGT>CTT & 0 & 0 \\
pGly12Ile; codon 12 GGT>ATT & 0 & 0 \\
pGly13Asp; codon 13 GGC>GAC & 5 & 11.4 \\
pGly13Cys; codon 13 GGC > TGT & 0 & 0 \\
\hline
\end{tabular}

$K R A S, \mathrm{v}-\mathrm{Ki}$-ras2 Kirsten rat sarcoma.

Table III. Correlation between KRAS mutational status and tumoral variables.

\begin{tabular}{|c|c|c|c|c|c|}
\hline \multirow[b]{2}{*}{ Characteristic } & \multicolumn{2}{|c|}{$\begin{array}{c}\text { Mutated } \\
K R A S\end{array}$} & \multicolumn{2}{|c|}{$\begin{array}{c}\text { wt } \\
K R A S\end{array}$} & \multirow[b]{2}{*}{ P-value } \\
\hline & No. & $\%$ & No. & $\%$ & \\
\hline Gender & & & & & 1.000 \\
\hline Female & 22 & 50 & 23 & 41 & \\
\hline Male & 22 & 50 & 33 & 59 & \\
\hline Age (years) & & & & & 1.000 \\
\hline$>60$ & 11 & 25 & 22 & 39 & \\
\hline$<60$ & 33 & 75 & 34 & 61 & \\
\hline Tumor location & & & & & 1.000 \\
\hline Colon, NOS & 58 & 58 & 20 & 67 & \\
\hline Rectum & 22 & 22 & 5 & 17 & \\
\hline Rectosigmoid & 20 & 20 & 5 & 16 & \\
\hline Primary tumor stage & & & & & 1.000 \\
\hline I & 0 & 0 & 0 & 0 & \\
\hline II & 4 & 9 & 3 & 5 & \\
\hline III & 5 & 11 & 11 & 20 & \\
\hline IV & 35 & 80 & 42 & 75 & \\
\hline
\end{tabular}

$K R A S$, v-Ki-ras2 Kirsten rat sarcoma; NOS, not otherwise specified.

and gene mutational status (Table IV). A complete response was observed in one patient out of 25 with wild-type $K R A S$. Patients (7) with partial responses included $3 K R A S$ wt and $4 K R A S$ mutants. A similar pattern was also observed for patients with stable or progressive disease. Of the patients with the mutated KRAS gene, $73 \%$ had progressive disease compared with $68 \%$ of patients with the wild-type gene.

Prevalence of KRAS mutations in Jordan and other countries. A review of studies published from various countries concerning the prevalence of KRAS mutations in colorectal tumors is 


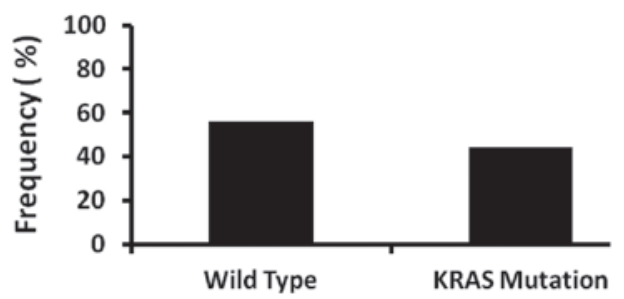

Figure 1. Frequency of KRAS mutations among Jordanian CRC patients. $K R A S, \mathrm{v}-\mathrm{Ki}$-ras2 Kirsten rat sarcoma.

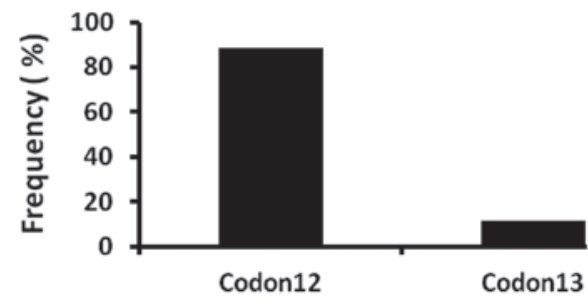

Figure 2. Mutational events in KRAS gene. KRAS codons 12 and 13 analysis in 100 patients. KRAS, v-Ki-ras2 Kirsten rat sarcoma.

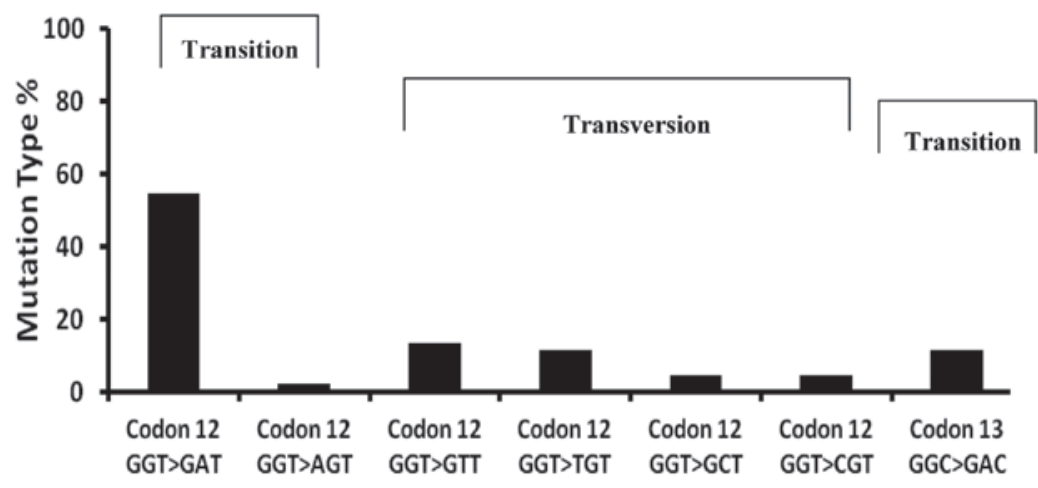

Figure 3. Distribution chart of tested KRAS mutations in the group of tested patients. The CRCs of Jordanian patients had more transitional mutations as compared with transversion mutations. KRAS, v-Ki-ras2 Kirsten rat sarcoma.

Table IV. Association of KRAS mutational status with treatment outcomes.

\begin{tabular}{|c|c|c|c|c|}
\hline \multirow[b]{2}{*}{ Response to therapy } & \multicolumn{2}{|c|}{$\begin{array}{c}\text { Mutated } \\
K R A S\end{array}$} & \multicolumn{2}{|c|}{$\begin{array}{c}\text { wt } \\
K R A S\end{array}$} \\
\hline & No. & $\%$ & No. & $\%$ \\
\hline Complete response & 0 & 0 & 1 & 4 \\
\hline Partial response & 4 & 15.5 & 3 & 12 \\
\hline Stable disease & 3 & 11.5 & 4 & 16 \\
\hline Progressive disease & 19 & 73 & 17 & 68 \\
\hline
\end{tabular}

$K R A S, \mathrm{v}-\mathrm{Ki}$-ras2 Kirsten rat sarcoma.

shown in Table V (16-21). In most countries, the KRAS mutation rate ranged from $18-47 \%$, as identified in Egypt and the United States, respectively. Thus, the prevalence rate identified in Jordan (44\%) is at the higher end of this range. In addition, the prevalence of KRAS mutations in Jordanian patients was markedly higher than its two closest neighbors, Saudi Arabia and Turkey (28 and $37.5 \%$, respectively). However, the fraction of mutations revealed in codon 12 and 13 of Jordanian CRC patients was similar to all other countries, with the exception of the United States.

\section{Discussion}

$\mathrm{CRC}$ is one of the leading causes of mortality due to cancer in Jordan. The poor prognosis of this disease would be amelio- rated if curative surgery was performed in its early stages. This study analyzed KRAS mutations in CRCs of Jordanian patients, in whom CRC incidence and mortality is one of the highest in the Middle East and is still increasing (2). The incidence of KRAS mutations in Jordan was $44 \%$ and these mutations were predominantly observed in codon $12(89 \%)$. These results, from a series of 100 patients, are in accordance with a modern series conducted worldwide. KRAS codon 12 and 13 mutations cover $98 \%$ of the entire KRAS mutation spectrum in CRC $(22,23)$. Thus, this analysis did not include other codons, including 61 (exon 3) or 146 (exon 4) due to their infrequency in CRC.

To assess the specificity of both the TheraScreen DxS $K R A S$ Mutation Kit and Vienna Lab methods used to analyze $K R A S$ status in this study, the concordance of test results was evaluated for retrospective samples with the results of the Sanger sequencing method. The real-time PCR results (DxS kit) and allele-specific oligonucleotide hybridization results (Vienna Lab) were in $100 \%$ concordance when compared with the Sanger sequencing method. A number of comparative studies have evaluated the performance of the various methods used to accurately characterize KRAS gene status $(24,25)$. The majority of these studies agree that the DxS and Vienna Lab kits are equivalent and more reliable due to their higher sensitivity than Sanger sequencing (24). The DxS kit tests for the seven most common mutations in codon 12 and 13 of KRAS and Vienna Lab detects 10 mutations. A significant number (44\%) of KRAS mutations were detected using these two kits which may account for a large number of mutations in the Jordanian population. Thus, the detection methods utilized in this study are considered to produce an accurate frequency of KRAS mutations. 
Table V. Prevalence of KRAS mutations in Jordan and other countries.

\begin{tabular}{|c|c|c|c|c|c|c|c|c|}
\hline & Jordan & Saudi Arabia & Egypt & USA & Brazil & Turkey & Spain & Slovenia \\
\hline KRAS mutated tumors $(\%)$ & 44 & 28 & 18 & 47 & 35 & 37.5 & 34 & 45.5 \\
\hline Mutated in codon $12(\%)$ & 89 & 81 & NA & 96 & 85 & 82 & 84 & 81 \\
\hline Mutated in codon $13(\%)$ & 11 & 19 & NA & 4 & 15 & 18 & 16 & 19 \\
\hline
\end{tabular}

$K R A S, \mathrm{v}-\mathrm{Ki}$-ras2 Kirsten rat sarcoma.

The frequency and spectrum of KRAS mutations did not differ when compared with those of most other studies (16-21). The distribution of the seven tested mutations (p.Gly12Asp, 54.5\%; p.Gly12Val, 13.6\%; p.Gly12Cys, $11.4 \%$; p.Gly12Ala, 4.5\%; p.Gly12Arg, 4.5\%; pGly12Ser, $2.3 \%$ and p.Gly13Asp, $11.4 \%$ ) among the mutated $K R A S$ patients is in accordance with published data (21). The findings of this study suggest that the frequency of KRAS mutations in Jordan is similar to those in European countries and the United States. However, Jordanian KRAS mutation data contrasted sharply with the neighboring countries of Saudi Arabia and Egypt. A study concerning KRAS mutation status in Saudi Arabia reported a significantly lower frequency (28\%) than its neighboring Jordan (19). An Egyptian study reported that mutations of the KRAS proto-oncogene is uncommon (18\%) in Egyptian CRC in contrast to Western cases and was not identified in any patients under the age of 40 years (26). Although Arabic countries share certain cultural background and environmental exposures, these findings reveal possible molecular genetic determinants playing a role in KRAS gene mutation. Thus, ethnicity and geographical differences should be considered in designing future clinical trials. Overall, the frequency and spectrum of KRAS mutations did not differ when compared to the majority of other reports, possibly due to the nature of mutations in the KRAS gene giving the tumor cell a growth advantage leading to clonal selection.

In this study, no statistically significant difference between $K R A S$ positivity rate and the clinicopathological findings was observed. Certain studies have reported a higher frequency of $K R A S$ mutations in females compared with males $(18,27)$. This study had an almost equal ratio of males to females but identified no statistical difference in KRAS mutation with respect to gender. Tumor stage revealed no correlation with mutation status, which is in agreement with published studies $(17,28)$.

The association between KRAS mutational status and prognosis remains controversial for patients with metastatic CRC that have not been treated with anti-EGFR antibodies. While certain studies reported a link between KRAS mutations and poor prognosis $(15,17)$, others have identified no association (29). The biggest clinical trial designed to analyze the prognostic value of KRAS status was the RASCAL study, which revealed that a glycine-to-valine mutation in codon 12 increased the risk of recurrence and mortality by $30 \%$, irrespective of the type of therapy administered (29). A smaller scale study from Spain published findings that agreed with the RASCAL study on the poor prognosis for patients with KRAS-mutated primary tumors. However, the Spanish group revealed contrasting results to the RASCAL study by declaring that the mutation type did not affect prognosis (17). One limitation in the current study was the unavailability of information concerning treatment outcomes since the study was in retrospect. Only 51 patients had available information concerning response to therapy documented in the KHCC Cancer Registry and of those only 26 were $K R A S$-mutated patients. Thus, we were unable to perform analysis to deduce whether an association existed among disease-free, progression-free or overall survival rates and KRAS mutational status or type. However, the available data for the group of patients with stable disease provide inconclusive evidence as the KRAS mutational status is negative for almost half of the patients. Additionally, among the 36 patients who were progressing and did not respond to therapy, 17 were $K R A S$-wt and 19 harbored $K R A S$ mutations. A similar pattern of results was also reported by Licar et al suggesting other molecular elements of response require identification (21).

Information from a larger scale future study in Jordan will either confirm or refute that the presence of activating mutations in the KRAS gene reveals a poor prognostic group and non-responding patients to anti-EGFR antibodies. Further investigation will be directed at how Food and Drug Administration agencies handle KRAS mutational analysis prior to targeted drug administration to CRC patients.

Increasing efforts are exerted to assess individual specific molecular alterations for personalized diagnosis, prognosis and/or treatment. Future studies should be of larger sizes and any existing concordance between $K R A S$ mutations of primary and metastatic tumors from patients with $\mathrm{CRC}$ requires identification. A number of previous studies have reported a high degree of concordance in KRAS mutational status between primary tumors and their related liver metastases (17,30-32). The liver and lung are common sites of CRC metastases, however, this study revealed liver metastases to be more common (70\%). Determining the degree of concordance is critical from three aspects. First, the frequency of KRAS mutations in primary and secondary tumors in patients needs to be compared with published studies to determine whether the acquired data are in agreement. Second, evidence is required to further support previous studies that KRAS mutations occur early in carcinogenesis (33). Third, evaluation of the mutational status of KRAS may be performed from a metastatic site in the case of a primary tumor sample being unavailable.

In summary, KRAS mutation and subtyping analysis of CRCs in Jordanian patients confirmed the data from other studies but also yielded potentially new recommendations. The results of this study will have a major impact on disease management as the cost of treating metastatic CRC will be 
reduced by millions of Jordanian Dinars a year, if all patients were tested for KRAS mutations. Using KRAS testing to restrict the use of EGFR-inhibitor therapy to patients with wild-type $K R A S$ tumors would avoid the administration of unnecessary, ineffective and toxic treatments to patients with KRAS mutations who would not benefit from them.

\section{Acknowledgements}

The authors would like to thank Selena Audeh, Roubi Abu Obeid, Firas Zaitar and Nadim Musleh for their kind assistance.

\section{References}

1. Jemal A, Siegel R, Ward E, Hao Y, Xu J, Murray T and Thun MJ: Cancer statistics, 2008. CA Cancer J Clin 58: 71-96, 2008.

2. Tarawneh M, Nimri O, Arkoob K and Al Zhagal M: Cancer Incidence in Jordan annual report, 2009.

3. Penna $\mathrm{C}$ and Nordlinger B: Colorectal metastasis (liver and lung). Surg Clin North Am 82: 1075-1090, 2002.

4. Van Krieken JH, Jung A, Kirchner T, et al: KRAS mutation testing for predicting response to anti-EGFR therapy for colorectal carcinoma: proposal for an European quality assurance program. Virchows Arch 453: 417-431, 2008.

5. Fransėn K, Klintenäs M, Osterström A, et al: Mutation analysis of the BRAF, ARAF and RAF-1 genes in human colorectal adenocarcinomas. Carcinogenesis 25: 527-533, 2004.

6. Jonker DJ, O'Callaghan CJ, Karapetis CS, et al: Cetuximab for the treatment of colorectal cancer. N Engl J Med 357: 2040-2048, 2007.

7. Van Cutsem E, Siena S, Humblet Y, et al: An open-label, singlearm study assessing safety and efficacy of panitumumab in patients with metastatic colorectal cancer refractory to standard chemotherapy. Ann Oncol 19: 92-98, 2008.

8. Amado RG, Wolf M, Peeters M, Van Cutsem E, et al: Wild-type KRAS is required for panitumumab efficacy in patients with metastatic colorectal cancer. J Clin Oncol 26: 1626-1634, 2008.

9. Khambata-Ford S, Garrett CR, Meropol NJ, et al: Expression of epiregulin and amphiregulin and K-ras mutation status predict disease control in metastatic colorectal cancer patients treated with cetuximab. J Clin Oncol 25: 3230-3237, 2007.

10. Karapetis CS, Khambata-Ford S, Jonker DJ, et al: K-ras mutations and benefit from cetuximab in advanced colorectal cancer. N Engl J Med 359: 1757-1765, 2008.

11. Zenker M, Lehmann K, Schulz AL, et al: Expansion of the genotypic and phenotypic spectrum in patients with KRAS germline mutations. J Med Genet 44: 131-135, 2007.

12. Wong $\mathrm{R}$ and Cunningham $\mathrm{D}$ : Using predictive biomarkers to select patients with advanced colorectal cancer for treatment with epidermal growth factor receptor antibodies. J Clin Oncol 26: 5668-5670, 2008.

13. Minamoto T, Mai M and Ronai Z: K-ras mutation: early detection in molecular diagnosis and risk assessment of colorectal, pancreas, and lung cancers - a review. Cancer Detect Prev 24: 1-12, 2000.

14. Calistri D, Rengucci C, Seymour I, et al: Mutation analysis of p53, K-ras, and BRAF genes in colorectal cancer progression. J Cell Physiol 204: 484-488, 2005.

15. Lièvre A, Bachet JB, Le Corre D, et al: KRAS mutation status is predictive of response to cetuximab therapy in colorectal cancer. Cancer Res 66: 3992-3995, 2006.
16. Edkins S, O'Meara S, Parker A, et al: Recurrent KRAS codon 146 mutations in human colorectal cancer. Cancer Biol Ther 5: 928-932, 2006

17. Cejas P, López-Gómez M, Aguayo C, et al: KRAS mutations in primary colorectal cancer tumors and related metastases: a potential role in prediction of lung metastasis. PLoS One 4: e8199, 2009.

18. Ferreira CG, Zalcberg-Renault I, Vieira FM, Bonamino MH and Zalis M: Analysis of KRAS mutations in colorectal cancer (CRC) patients by gender in a Brazilian cohort of 3,346 patients. J Clin Oncol 28: 15s, 2010.

19. Abubaker J, Bavi P, Al-Haqawi W, et al: Prognostic significance of alterations in KRAS isoforms KRAS-4A/4B and KRAS mutations in colorectal carcinoma. J Pathol 219: 435-445, 2009.

20. Conzelmann M, Linnemann U and Berger MR: K-ras codon 12 and 13 mutations are correlated with differential patterns of tumor cell dissemination in colorectal cancer patients. Int $\mathrm{J}$ Oncol 24: 1537-1544, 2004.

21. Licar A, Cerkovnik P, Ocvirk J and Novakovic S: KRAS mutations in Slovene patients with colorectal cancer: frequency, distribution and correlation with the response to treatment. Int J Oncol 36: 1137-1144, 2010.

22. Fearon ER: Molecular genetic studies of the adenoma-carcinoma sequence. Adv Intern Med 39: 123-147, 1994.

23. Shaw P, Tardy S, Benito E, et al: Occurrence of Ki-ras and p53 mutations in primary colorectal tumors. Oncogene 6: 2121-2128, 1991.

24. Herreros-Villanueva M and Aggarwal G: KRAS assay selection: sensitivity and accuracy in clinical application. Mol Biol Rep 39: 2467-2470, 2012

25. Pinto P, Rocha P, Veiga I, et al: Comparison of methodologies for KRAS mutation detection in metastatic colorectal cancer. Cancer Genet 204: 439-46, 2011.

26. Soliman AS, Bondy ML, El-Badawy SA, et al: Contrasting molecular pathology of colorectal carcinoma in Egyptian and Western patients. Br J Cancer 85: 1037-1046, 2001.

27. Nagasaka T, Sasamoto H, Notohara K, et al: Colorectal cancer with mutation in BRAF, KRAS, and wild-type with respect to both oncogenes showing different patterns of DNA methylation. J Clin Oncol 22: 4584-4594, 2004.

28. Sharma N, Saifo M, Tamaskar I, Bhuvaneswari R, Mashtare T and Fakih M: KRAS status and clinical outcome in metastatic colorectal cancer patients treated with first-line FOLFOX chemotherapy. J Gastrointest Oncol 1: 90-96, 2010.

29. Andreyev HJ, Norman AR, Cunningham D, et al: Kirsten ras mutations in patients with colorectal cancer: the 'RASCAL II' study. Br J Cancer 85: 692-696, 2001.

30. Santini D, Loupakis F, Vincenzi B, et al: High concordance of KRAS status between primary colorectal tumors and related metastatic sites: implications for clinical practice. Oncologist 13: 1270-1275, 2008

31. Etienne-Grimaldi MC, Formento JL, Francoual M, et al: K-Ras mutations and treatment outcome in colorectal cancer patients receiving exclusive fluoropyrimidine therapy. Clin Cancer Res 14: 4830-4835, 2008.

32. Artale S, Sartore-Bianchi A, Veronese SM, et al: Mutations of KRAS and BRAF in primary and matched metastatic sites of colorectal cancer. J Clin Oncol 26: 4217-4219, 2008.

33. Vogelstein B, Fearon ER, Hamilton SR, et al: Genetic alterations during colorectal-tumor development. N Engl J Med 319 : $525-532,1988$. 